\title{
Normalization of a Poisson algebra is Poisson
}

\author{
D. Kaledin* \\ Steklov Math Institute
}

\section{Introduction}

It is well-known that functions on a smooth symplectic manifold $M$ are equipped with a canonical skew-linear operation called the Poisson bracket. The bracket is compatible with multiplication in a certain precise way. Formalizing this structure, one obtains the notion of a Poisson algebra (see Definition 1.11).

The definition of a Poisson algebra is quite general; among other things, it involves no assumption of smoothness. Recently there appeared good reasons to study Poisson algebras in full generality. In particular, they seem to be quite useful in the study of the so-called symplectic singularities initiated by A. Beauville [B].

However, while non-trivial Poisson structures on smooth manifolds have been under close scrutiny for fifty years or more, the general theory is much less developed. It seems that even the simplest facts are not known, or at least, not easy to find in the existing literature.

The goal of the present note is to prove one of these simple facts - namely, we prove that the integral closure of a Poisson algebra is again Poisson (Theorem [1.5). The exposition is essentially self-contained. We need a couple of preliminary lemmas which are definitely not new, but not quite standard, either. To spare the reader the trouble of extensive book search, we have taken the liberty of re-proving them from scratch.

\section{Statements and definitions.}

Fix once and for all a base field $k$ of characteristic char $k=0$.

Definition 1.1. A Poisson algebra over the field $k$ is a commutative algebra $A$ over $k$ equipped with an additional skew-linear operation $\{-,-\}: A \otimes A \rightarrow A$ such that

$\underline{(1.1)}\{a, b c\}=\{a, b\} c+\{a, c\} b \quad, \quad 0=\{a,\{b, c\}\}+\{b,\{c, a\}\}+\{c,\{a, b\}\}$,

\footnotetext{
${ }^{*}$ Partially supported by CRDF grant RM1-2354-MO-02.
} 
for all $a, b, c \in A$. An ideal $I \subset A$ is called a Poisson ideal if $\{i, a\} \in I$ for any $i \in I, a \in A$.

Additionally, we will always assume that a Poisson algebra $A$ has a unit element $1 \in A$ such that $\{1, a\}=0$ for every $a \in A$.

Definition 1.2. A Poisson scheme over $k$ is a scheme $X$ over $k$ equipped with a skew-linear bracket in the structure sheaf $\mathcal{O}_{X}$ satisfying (1.1).

Lemma 1.3. Let $A$ be a Poisson algebra.

(i) For any multiplicative system $S \subset A$, the localization $A\left[S^{-1}\right]$ carries a canonical Poisson algebra structure.

(ii) Any associated prime ideal $\mathfrak{p} \subset A$ is a Poisson ideal.

(iii) The radical $J \subset A$ of the algebra $A$ is a Poisson ideal.

Proof. For (i), set

$$
\begin{aligned}
\left\{a_{1} s_{1}^{-1}, a_{2} s_{2}^{-1}\right\}= & \left\{a_{1}, a_{2}\right\}\left(s_{1} s_{2}\right)^{-1}-\left\{a_{1}, s_{2}\right\} a_{2}\left(s_{1} s_{2}^{2}\right)^{-1} \\
& -\left\{s_{1}, a_{2}\right\} a_{1}\left(s_{1}^{2} s_{2}\right)^{-1}+a_{1} a_{2}\left\{s_{1}, s_{2}\right\}\left(s_{1}^{2} s_{2}^{2}\right)^{-1} .
\end{aligned}
$$

For (ii), note that $\mathfrak{p} \subset A$ is the kernel of the canonical Poisson map from the Poisson algebra $A$ to the fraction field $A_{\mathfrak{p}}$. For (iii), note that $J$ is the intersection of all the associated primes.

Lemma 1.3 (i), in particular, means that the spectrum of a Poisson algebra is a Poisson scheme. We also note the following geometric corollary.

Corollary 1.4. Let $X$ be a Poisson scheme over $k$. Then the reduction $X_{\text {red }}$ of the scheme $X$ is a Poisson scheme, and so is every irreducible component $X_{0}$ of the reduction $X_{\text {red }}$.

Our main result is the following.

Theorem 1.5. Let $A_{0}$ be a Noetherian domain over $k$, and let $A$ be its integral closure in its fraction field.

(i) Every derivation $\xi$ of the algebra $A_{0}$ extends to a derivation of the algebra A.

(ii) Every Poisson bracket $\{-,-\}$ on the algebra $A_{0}$ extends to a Poisson bracket on the algebra $A$.

Note that both derivations and Poisson brackets extend naturally and uniquely to the fraction field $\operatorname{Frac} A_{0}=\operatorname{Frac} A$. The point is that both preserve the integral closure $A \subset$ Frac $A$. The first claim is well-known; nevertheless, we will prove it, because it is needed in the proof of (ii).

The geometric corollary (in fact, an equivalent geometric formulation) of Theorem 1.5 is the following. 
Corollary 1.6. Let $X_{0}$ be a Noetherian integral scheme over $k$, and let $X$ be its normalization. Then every vector field $\xi$ on $X_{0}$ and every Poisson scheme structure on $X_{0}$ extend to $X$.

\section{Discrete valuation rings.}

To prove Theorem 1.5 we first study the situation in codimension 1 . In this section, assume given a local Noetherian algebra $A_{0}$ over $k$ of dimension 1 . Let $K_{0}$ be its residue field. Let $A$ be the integral closure of the algebra $A_{0}$. It is well-known that $A$ is a discrete valuation ring, whose residue field $K$ is a finite extension of the residue field $K_{0}$. Denote the valuation by $v$. Fix a uniformizing element $\pi \in A, v(\pi)=1$.

Lemma 2.1. There exists a single element $x \in A$ generating $A$ over $A_{0}$.

Proof. By the Primitive Element Theorem, the field $K$ is generated over $K_{0}$ by a single element, say $\bar{x}$. Let $P(x)$ be the minimal polynomial for $\bar{x}$ over $K_{0}$. Lift $\bar{x}$ to an element $x \in A$ and consider

$$
y=P(x) \in A .
$$

By definition, we have $y=0 \bmod \pi$, so that $v(y)>0$. If $v(y)=1$, we are done: $x$ and $y$ generate $A$ over $A_{0}$, and $y=P(x)$. If not, replace $y$ with

$$
y^{\prime}=P(x+\pi) .
$$

By the binomial formula, we have

$$
y^{\prime}=P^{\prime}(\bar{x}) \pi \quad \bmod \pi^{2} .
$$

Since the polynomial $P$ is minimal, its derivative $P^{\prime}$ satisfies $P^{\prime}(\bar{x}) \neq 0$. Therefore $v\left(y^{\prime}\right)=1$, and we are done: $A$ is generated over $A_{0}$ by $x+\pi$.

Lemma 2.2. Every derivation $\xi_{0}: A_{0} \rightarrow A_{0}$ of the algebra $A_{0}$ extends to a derivation of the algebra $A$.

Proof. Consider the formal power series algebra $B=A[[t]]$ in one indeterminate $t$. It is a regular local algebra, in particular, it is integrally closed (see, for example, $[\mathrm{AC}$, Prop. 14]). Therefore it coincides with the integral closure of the power series algebra $B_{0}=A_{0}[[t]]$. By functoriality, every automorphism of the algebra $B_{0}$ extends to an automorphism of its integral closure $B$. Consider the automorphism $\sigma_{0}: B_{0} \rightarrow B_{0}$ given by

$$
\sigma_{0}(t)=t \quad \sigma_{0}(a)=\exp (t \xi)(a) \text { for } a \in A_{0} \subset B_{0} .
$$

Extend it to an automorphism $\sigma: B \rightarrow B$ of the algebra $B$. Setting

$$
\xi(a)=\frac{\partial}{\partial t} \sigma(a) \bmod t
$$


gives a derivation $\xi: A \rightarrow A=B / t B$ extending the given derivation $\xi_{0}$.

Lemma 2.3. Assume that the algebra $A_{0}$ is equipped with a Poisson bracket $\{-,-\}$. Then this bracket extends uniquely to the algebra $A$.

Proof. Extend the bracket to the fraction field Frac $A$. We have to prove that $\{f, g\} \in A$ for every $f, g \in A$. Let $x \in A$ be the generator provided by Lemma 2.1 It suffices to prove that $\{x, x\} \in A$ and $\{x, f\} \in A$ for every $f \in A_{0}$. But $\{x, x\}=0$ tautologically, and $\{x, f\} \in A$ by Lemma 2.2 (define $\xi_{0}: A_{0} \rightarrow A_{0}$ by $\left.\xi_{0}(a)=\{a, f\}\right)$.

\section{Proof of the Theorem.}

We can now prove Theorem 1.5 It is more convenient to approach it in the geometric form of the Corollary 1.6 Thus, let $X_{0}$ be a Noetherian integral scheme, and let $X$ be its normalization. By Lemma 2.2 and Lemma 2.3 Corollary 1.6 holds for the open complement $U \subset X$ to a subscheme $Z \subset X$ of codimension $\operatorname{codim} Z \geq 2$. Therefore we have a derivation and/or a Poisson bracket on the structure sheaf $\mathcal{O}_{U}$. This induces a derivation and/or a Poisson bracket on the sheaf $j_{*} \mathcal{O}_{U}$, where $j: U \hookrightarrow X$ is the embedding. Since codim $Z \geq 2$, and $X$ is normal, we have $\mathcal{O}_{X} \cong j_{*} \mathcal{O}_{U}$.

Acknowledgements. I would like to thank E. Amerik, R. Bezrukavnikov, A. Kuznetsov, M. Rovinsky and M. Verbitsky for stimulating discussions.

\section{References}

[AC] N. Bourbaki, Algèbgre Commutative, Ch. 5, Hermann, Paris, 1964.

[B] A. Beauville, Symplectic singularities, Invent. Math. 139 (2000), 541-549.

Steklov Math Institute

Moscow, USSR

E-mail: kaledin@mccme.ru 